\title{
Radiation Enteritis
}

National Cancer Institute

\section{Source}

National Cancer Institute. Radiation Enteritis. NCI Thesaurus. Code C3905.

Inflammation of the small intestine caused by radiation exposure. 